\title{
DETECTION OF ADULTERATION OF EXTRA VIRGIN OLIVE OILS AVAILABLE ON THE POLISH MARKET
}

\author{
WYKRYWANIE ZAFAŁSZOWAŃ OLIWY Z OLIWEK EXTRA VIRGIN \\ DOSTĘPNEJ NA RYNKU POLSKIM
}

\begin{abstract}
Summary
Background. Extra virgin olive oils are valuable and healthy products, appreciated by consumers. The aim of this study was an attempt to detect adulteration of olive oils available on the Polish market (derived from Greece, Spain, and Italy) according to European Union Commission Regulation No 1348/2013.

Material and methods. Ten extra virgin olive oils available on the Polish market were analysed for fatty acids composition, and amount and composition of sterols (including erythrodiol and uvaol) using gas chromatography coupled with a mass spectrometry, and values of extinction coefficients $\left(\mathrm{K}_{232}\right.$ and $\left.\mathrm{K}_{270}\right)$. The results were compared with the requirements of European Union Commission Regulation No 1348/2013.

Results. One of the most efficient methods for detecting olive oil adulteration is qualitative and quantitative analysis of sterols. In certain of the analysed extra virgin olive oils some abnormalities in composition were detected, which may indicate an attempt to falsify the products. One of the methods can detect if extra virgin olive oils have been adulterated, and if they were falsified with the addition of olive pomace oil.

Conclusions. Our research proves that consumers are sometimes misinformed and some food manufacturers attempt to sell lower quality products as high-quality products. There is a need to constantly monitor the quality of foodstuffs and provide consumers with access to reliable information on food products.
\end{abstract}

Key words: olive oil, fatty acids, sterols, extinction coefficient, adulteration 
Derewiaka, D., Łuczak, A., Reder, M. (2016). Detection of adulteration of extra virgin olive oils available on the Polish market. Nauka Przyr. Technol., 10, 4, \#47. DOI: http://dx.doi.org/10.17306/J.NPT.2016.4.47

\section{Introduction}

Extra virgin olive oil is the most valuable among all types of olive oils (virgin olive oil, lampante virgin olive oil and also refined olive oil). It has many beneficial nutrients (such as tocopherols, polyphenols, squalene), as well as the highest quality. EVOO (extra virgin olive oil) is extracted from olive fruits either by mechanical or other physical means (e.g. by centrifugation) and it is not subjected to the refining process. Due to those processes the composition of olive oil is not altered and gives a specific smell, delicate flavour and a slightly greenish tinge. Olive pomace oil has lower quality and is produced from the pomace oil by extraction with organic solvents and enriched by addition of virgin olive oil. From the dietary point of view extra virgin olive oil is the most valuable for human consumption. EVOO can be falsified mixing it with other vegetable oils or by adding a lower quality olive oil (Garcia et al., 2013). In order to protect and ensure the quality of EVOO and its authenticity, the EU introduced Commission Implementing Regulations No 299/2013 and No 1348/2013 (Commission..., 2013a, 2013b) amending the Commission Regulation No 2568/91 on the characteristics of olive oil and olive-residue oil and the relevant methods of analysis, which recommended for instance fatty acid profile, sterol concentration and analysis of values of extinction coefficients as parameters to investigate olive oil adulteration.

Detecting adulteration of EVOO by addition of other oils can be performed by checking the composition of triacylglycerols (TAGs) and fatty acids (FA). Falsification of EVOO by addition of VOO (virgin olive oil), LVOO (lampante virgin olive oil), ROO (refined olive oil) is mostly based on some target compounds which belong to olive oil's bioactive compounds, namely chlorophylls, diacylglycerols, esters of FA, straight chain wax esters, sterol components and also TAGs. Methods used to determine the above mentioned parameters, which are recommended by Commission Regulation (EEC) No 2568/91... (1991), are as follows: HPLC, GC, TLC, SPE, spectrophotometric and organoleptic. There is a growing interest in exploring an alternative methodology based on the application like DNA methods, electronic nose and others to detect EVOO adulteration (Aparicio et al., 2013; Garcia et al., 2013).

The aim of the study was to check the authenticity of EVOO's present on the Polish and European market.

\section{Material and methods}

Samples of extra virgin olive oil (derived from Greece - product No 1, Spain products Nos, 2, 4, 9, 10, and Italy - Nos. 3, 5, 6, 7, 8) and olive pomace oil (No 11 derived from Spain) were purchased in Polish supermarkets in 2012 and 2013.

\section{Determination of fatty acids composition by GC-MS}

$50 \mathrm{mg}$ of olive oil was dissolved in $2 \mathrm{ml}$ of hexane, then vortexed vigorously for $1 \mathrm{~min}$ to achieve sample uniformity and $20 \mu \mathrm{l}$ of the mixture was taken. Transesterification of the samples was conducted at room temperature for $1 \mathrm{~h}$ by adding $0.5 \mathrm{ml} 2 \mathrm{M}$ $\mathrm{KOH}$ solution in methanol (with periodic shaking) and $2 \mathrm{ml}$ of hexane was added. The 
Derewiaka, D., Łuczak, A., Reder, M. (2016). Detection of adulteration of extra virgin olive oils available on the Polish market. Nauka Przyr. Technol., 10, 4, \#47. DOI: http://dx.doi.org/10.17306/J.NPT.2016.4.47

hexane layer $(1 \mathrm{ml})$ was transferred into a glass ampoule and $0.5 \mathrm{ml}$ of hexane was added. ZB FFAP $(30 \mathrm{~m} \times 0.25 \mathrm{~mm} \times 0.25 \mu \mathrm{m})$ capillary column was used to separate fatty acid methyl esters with helium as a carrier gas, at a flow rate of $0.60 \mathrm{ml} / \mathrm{min}$. The injector temperature was $230^{\circ} \mathrm{C}$, and the column temperature was programmed as follows: $60^{\circ} \mathrm{C}$ for $4 \mathrm{~min}$, subsequent increase to $230^{\circ} \mathrm{C}$ at the rate of $4^{\circ} \mathrm{C} / \mathrm{min}$ and maintained for $10 \mathrm{~min}$. The interface temperature for GC-MS was $225^{\circ} \mathrm{C}$. The temperature of ion source was $200^{\circ} \mathrm{C}$, ionization energy was $70 \mathrm{~V}$. The split ratio was $20: 1$. The total ion control (TIC) was used to detect fatty acids ( $\mathrm{m} / \mathrm{z}$ ranged from 50 to 400 ). Identification of fatty acids was made on the basis of mass spectral libraries (NIST 47, NIST 147 and Wiley 175), as well as data from literature, and by comparison of their retention times with the authentic standards GLC-85 and FAME Mix GLC-90. The results were expressed as $\mathrm{w} / \mathrm{w}(\%)$ total fatty acid. Three replicates per each sample were analysed.

\section{Determination of sterols concentration by GC-MS}

The internal standard $5 \alpha$-cholestane $(100 \mu \mathrm{l} ; 0.4 \mathrm{mg} / \mathrm{ml})$ was added to $150 \mathrm{mg}$ of olive oil and was dissolved in $2 \mathrm{ml}$ of hexane, then stirred for $1 \mathrm{~min}$. Transesterification of the samples was conducted at room temperature for $1-2 \mathrm{~h}$ by adding $0.5 \mathrm{ml} 2 \mathrm{M}$ $\mathrm{KOH}$ solution in methanol (with periodic shaking). An aliquot $(0.7 \mathrm{ml})$ of the hexane layer was transferred into a test tube and the solvent was evaporated under the nitrogen. The residue was dissolved in $100 \mu \mathrm{l}$ of pyridine and $100 \mu \mathrm{l}$,O-bis(trimethylsilyl)trifluoroacetamide with $1 \%$ trimethylchlorosilane and stored in the dark for $24 \mathrm{~h}$ to complete derivatisation. Then, $1 \mathrm{ml}$ of hexane was added and $1 \mu \mathrm{l}$ of the mixture was collected for GC-MS analysis.

A ZB-5 ms $(30 \mathrm{~m} \times 0.25 \mathrm{~mm} \times 0.25 \mu \mathrm{m})$ capillary column was used to separate sterols with helium as a carrier gas, at a flow rate of $1.18 \mathrm{ml} / \mathrm{min}$. The injector temperature was $275^{\circ} \mathrm{C}$, and the column temperature was programmed as follows: $65^{\circ} \mathrm{C}$ for 2 min, subsequent increase to $250^{\circ} \mathrm{C}$ at the rate of $5^{\circ} \mathrm{C} / \mathrm{min}$, then to $310^{\circ} \mathrm{C}$ at the rate of $3^{\circ} \mathrm{C} / \mathrm{min}$ for $10 \mathrm{~min}$. The interface temperature for GC-MS was $260^{\circ} \mathrm{C}$. The temperature of ion source was $250^{\circ} \mathrm{C}$, ionization energy was $70 \mathrm{~V}$. The total ion control (TIC) was used to detect sterols ( $\mathrm{m} / \mathrm{z}$ ranged between 100-600). The internal standard $5 \alpha$-cholestane was used to quantify trimethylsilyl (TMS) derivatives of sterols. Identification of cholesterol was made on the basis of mass spectral libraries (NIST 47, NIST 147 and Wiley 175), as well as data from literature, and by comparison of the retention time with authentic standard of cholesterol (Sigma-Aldrich Chemie GmbH, Germany). Three replicates per each sample were analysed.

\section{Determination of specific extinction at $232,270 \mathrm{~nm}$ and $\Delta K$}

About $0.25 \mathrm{~g}$ of the filtered sample was weighted in a volumetric flask $(25 \mathrm{ml})$ and solved in hexane. Next, a measurement of ultraviolet absorbance at 232 and $270 \mathrm{~nm}$ was performed using Shimadzu UV 160 UV-VIS Spectrophotometer apparatus. Three replicates per each sample were analysed.

Determination of the appropriate optical density (extinction coefficients) at different wavelengths was carried out: 
Derewiaka, D., Łuczak, A., Reder, M. (2016). Detection of adulteration of extra virgin olive oils available on the Polish market. Nauka Przyr. Technol., 10, 4, \#47. DOI: http://dx.doi.org/10.17306/J.NPT.2016.4.47

$$
\mathrm{K}_{\lambda}=\frac{\mathrm{E}_{\lambda}}{\mathrm{c} \times \mathrm{s}}
$$

where:

$\mathrm{K}_{\lambda}$ - the specific optical density at a wavelength of $\lambda$,

$\mathrm{E}_{\lambda}$ - the optical density at a wavelength of $\lambda$,

$\mathrm{c}-$ concentration of the solution per $100 \mathrm{ml}(\mathrm{g})$,

$\mathrm{s}-$ the thickness of the glass cuvette $(\mathrm{cm})$.

Determination of $\Delta K$ :

$$
\Delta \mathrm{K}=\mathrm{K}_{\mathrm{m}}-\frac{\mathrm{K}_{\mathrm{m}-4}+\mathrm{K}_{\mathrm{m}+4}}{2}
$$

where:

$\mathrm{K}_{\mathrm{m}}$ - the specific optical density of the wavelength (m).

\section{Data analysis}

The results were statistically analysed using STATISTICA 8.0 software. To determine the significance of the differences between the mean samples Tukey's test was used, at the significance level of $\mathrm{p}=0.05$.

\section{Results and discussion}

\section{Fatty acids composition}

In ten EVOO's (Nos. 1-10) and one olive pomace oil (No 11) 13 different fatty acids were determined (Table 1). The most abundant fatty acid in all the analysed olive oil samples was oleic acid and it ranged from 66.52 to $77.08 \%$. According to the Regulation No 1348/2013 (Commission..., 2013b) the limit of this acid should be between 55.00-83.00 for EVOO. Palmitic and linoleic fatty acids content ranged between 11.33$15.02 \%$ and $4.73-12.45 \%$, respectively. Limits of the palmitic and linoleic acid in EVOO according to Regulation No 1348/2013 (Commission..., 2013b) were 7.50 $20.00 \%$ and $3.50-21.00 \%$, respectively. The sum of the rest of fatty acids determined in EVOO and olive pomace oil did not exceed 6\%. Content of palmitoleic fatty acid ranged from 0.72 to $1.56 \%$ in all the investigated samples and did not exceed the requirements of the Regulation No 1348/2013 (Commission..., 2013b) - 3.50\%. The content of stearic acid in olives samples was between $2.51-3.75 \%$ and was lower than limitation presented in the Regulation $-5.00 \%$. In EVOO linoleic fatty acid content was between $0.56-0.63 \%$ and in olive pomace $-0.34 \%$ and it should be lower than $1 \%$. Van Wetten et al. (2015) in their studies found a similar percentage of fatty acids. In their studies, the percentage of oleic acid in EVOO was between $64.9-78.5 \%$, linoleic acid $5.7-12.8 \%$ and palmitic acid $-0.6-1.8 \%$. Results analogous to these presented in our study were obtained by Christopoulou et al. (2004). They found that the percentage of arachidonic acid in EVOO was $0.48 \%$, behenic acid $-0.15 \%$ and lignoceric acid did not exceed $0.06 \%$. Also Krichène et al. (2010) found that linolenic acid content in Tunisian olive oils ranged between $0.59-0.79 \%$. 
Derewiaka, D., Łuczak, A., Reder, M. (2016). Detection of adulteration of extra virgin olive oils available on the Polish market. Nauka Przyr. Technol., 10, 4, \#47. DOI: http://dx.doi.org/10.17306/J.NPT.2016.4.47

Table 1. Fatty acids composition in selected olive oils (\%)

Tabela 1. Skład kwasów tłuszczowych w wybranych oliwach z oliwek (\%)

\begin{tabular}{|c|c|c|c|c|c|c|c|c|c|c|c|c|c|}
\hline $\begin{array}{l}\text { Product } \\
\text { code } \\
\text { Kod } \\
\text { produktu }\end{array}$ & C $14: 0$ & C $16: 0$ & $\begin{array}{l}\text { C } 16: 1 \\
\text { (cis-9) }\end{array}$ & C $17: 0$ & $\begin{array}{l}\text { C 17:1 } \\
\text { (cis-9) }\end{array}$ & C 18:0 & $\begin{array}{l}\text { C 18:1 } \\
\text { (cis-9) }\end{array}$ & $\begin{array}{c}\text { C } 18: 2 \\
\text { (cis,cis- } \\
-9,12)\end{array}$ & $\begin{array}{c}\text { C } 18: 3 \\
\text { (cis,cis, } \\
\text { cis- } \\
-9,12,15 \text { ) }\end{array}$ & C $20: 0$ & $\begin{array}{l}\text { C 20:1 } \\
\text { (cis-9) }\end{array}$ & C 22:0 & C 24:0 \\
\hline 1 & $\begin{array}{c}0.02 \\
\pm 0.01^{\mathrm{a}}\end{array}$ & $\begin{array}{l}12.72 \\
\pm 0.00^{\mathrm{ab}}\end{array}$ & $\begin{array}{c}1.11 \\
\pm 0.18^{\mathrm{c}}\end{array}$ & $\begin{array}{c}0.05 \\
\pm 0.01^{\mathrm{a}}\end{array}$ & $\begin{array}{c}0.06 \\
\pm 0.02^{\mathrm{c}}\end{array}$ & $\begin{array}{c}2.91 \\
\pm 0.06^{\mathrm{ab}}\end{array}$ & \begin{tabular}{|l|}
74.74 \\
$\pm 0.02^{\text {acd }}$
\end{tabular} & $\begin{array}{c}6.94 \\
\pm 0.09^{\mathrm{ab}}\end{array}$ & $\begin{array}{c}0.57 \\
\pm 0.02^{\mathrm{ab}}\end{array}$ & $\begin{array}{c}0.44 \\
\pm 0.01^{\mathrm{abc}}\end{array}$ & $\begin{array}{c}0.28 \\
\pm 0.03^{\mathrm{abc}}\end{array}$ & $\begin{array}{c}0.16 \\
\pm 0.01^{\mathrm{ab}}\end{array}$ & $\begin{array}{c}0.03 \\
\pm 0.02^{\mathrm{a}}\end{array}$ \\
\hline 2 & $\begin{array}{c}0.01 \\
\pm 0.00^{\mathrm{a}}\end{array}$ & $\begin{array}{l}12.07 \\
\pm 0.29^{\text {ef }}\end{array}$ & $\begin{array}{c}1.02 \\
\pm 0.04^{\mathrm{abc}}\end{array}$ & $\begin{array}{c}0.06 \\
\pm 0.00^{\mathrm{ab}}\end{array}$ & $\begin{array}{c}0.09 \\
\pm 0.01^{\mathrm{a}}\end{array}$ & $\begin{array}{c}3.66 \\
\pm 0.12^{\mathrm{cd}}\end{array}$ & $\begin{array}{l}75.28 \\
\pm 0.66^{\mathrm{cd}}\end{array}$ & $\begin{array}{c}6.42 \\
\pm 0.22^{\mathrm{a}}\end{array}$ & $\begin{array}{c}0.61 \\
\pm 0.04^{\mathrm{a}}\end{array}$ & $\begin{array}{c}0.40 \\
\pm 0.02^{\mathrm{ab}}\end{array}$ & $\begin{array}{c}0.24 \\
\pm 0.01^{\mathrm{a}}\end{array}$ & $\begin{array}{c}0.11 \\
\pm 0.02^{\mathrm{a}}\end{array}$ & $\begin{array}{c}0.02 \\
\pm 0.01^{\mathrm{a}}\end{array}$ \\
\hline 3 & $\begin{array}{c}0.01 \\
\pm 0.00^{\mathrm{a}}\end{array}$ & $\begin{array}{l}12.54 \\
\pm 0.02^{\text {af }}\end{array}$ & $\begin{array}{c}1.05 \\
\pm 0.02^{\mathrm{bc}}\end{array}$ & $\begin{array}{c}0.08 \\
\pm 0.00^{\mathrm{abc}}\end{array}$ & $\begin{array}{c}0.14 \\
\pm 0.00^{\mathrm{b}}\end{array}$ & $\begin{array}{c}3.26 \\
\pm 0.01^{\text {acd }}\end{array}$ & \begin{tabular}{|l|}
74.78 \\
$\pm 0.07^{\text {acd }}$
\end{tabular} & $\begin{array}{c}6.75 \\
\pm 0.06^{\mathrm{a}}\end{array}$ & $\begin{array}{c}0.57 \\
\pm 0.01^{\mathrm{ab}}\end{array}$ & $\begin{array}{c}0.42 \\
\pm 0.00^{\mathrm{ab}}\end{array}$ & $\begin{array}{c}0.26 \\
\pm 0.01^{\mathrm{ab}}\end{array}$ & $\begin{array}{c}0.13 \\
\pm 0.01^{\mathrm{a}}\end{array}$ & $\begin{array}{c}0.01 \\
\pm 0.00^{\mathrm{a}}\end{array}$ \\
\hline 4 & $\begin{array}{c}0.01 \\
\pm 0.00^{\mathrm{a}}\end{array}$ & $\begin{array}{l}13.01 \\
\pm 0.08^{\mathrm{ab}}\end{array}$ & $\begin{array}{c}0.98 \\
\pm 0.04^{\mathrm{abc}}\end{array}$ & $\begin{array}{c}0.06 \\
\pm 0.00^{\mathrm{ab}}\end{array}$ & $\begin{array}{c}0.10 \\
\pm 0.00^{\mathrm{a}}\end{array}$ & $\begin{array}{c}2.87 \\
\pm 0.04^{\mathrm{ab}}\end{array}$ & $\begin{array}{l}73.32 \\
\pm 0.15^{\mathrm{b}}\end{array}$ & $\begin{array}{c}8.10 \\
\pm 0.05^{\mathrm{c}}\end{array}$ & $\begin{array}{c}0.60 \\
\pm 0.01^{\mathrm{a}}\end{array}$ & $\begin{array}{c}0.46 \\
\pm 0.00^{\mathrm{abc}}\end{array}$ & $\begin{array}{c}0.31 \\
\pm 0.00^{\mathrm{bc}}\end{array}$ & $\begin{array}{c}0.16 \\
\pm 0.00^{\mathrm{ab}}\end{array}$ & $\begin{array}{c}0.02 \\
\pm 0.00^{\mathrm{a}}\end{array}$ \\
\hline 5 & $\begin{array}{c}0.01 \\
\pm 0.00^{\mathrm{a}}\end{array}$ & $\begin{array}{l}12.82 \\
\pm 0.16^{\mathrm{ab}}\end{array}$ & $\begin{array}{c}0.97 \\
\pm 0.09^{\mathrm{abc}}\end{array}$ & $\begin{array}{c}0.07 \\
\pm 0.00^{\mathrm{ab}}\end{array}$ & $\begin{array}{c}0.12 \\
\pm 0.01^{\mathrm{a}}\end{array}$ & $\begin{array}{c}3.16 \\
\pm 0.47^{\mathrm{ac}}\end{array}$ & $\begin{array}{l}74.40 \\
\pm 0.15^{\mathrm{abc}}\end{array}$ & $\begin{array}{c}6.96 \\
\pm 0.55^{\mathrm{ab}}\end{array}$ & $\begin{array}{c}0.60 \\
\pm 0.01^{\mathrm{a}}\end{array}$ & $\begin{array}{c}0.43 \\
\pm 0.00^{\mathrm{abc}}\end{array}$ & $\begin{array}{c}0.29 \\
\pm 0.03^{\mathrm{abc}}\end{array}$ & $\begin{array}{c}0.14 \\
\pm 0.00^{\mathrm{a}}\end{array}$ & $\begin{array}{c}0.02 \\
\pm 0.00^{\mathrm{a}}\end{array}$ \\
\hline 6 & $\begin{array}{c}0.01 \\
\pm 0.01^{\mathrm{a}}\end{array}$ & $\begin{array}{l}13.09 \\
\pm 0.23^{\mathrm{b}}\end{array}$ & $\begin{array}{c}0.87 \\
\pm 0.04^{\mathrm{abc}}\end{array}$ & $\begin{array}{c}0.06 \\
\pm 0.00^{\mathrm{abc}}\end{array}$ & $\begin{array}{c}0.10 \\
\pm 0.00^{\mathrm{a}}\end{array}$ & $\begin{array}{c}2.52 \\
\pm 0.02^{\mathrm{b}}\end{array}$ & \begin{tabular}{|l|}
73.94 \\
$\pm 0.41^{\mathrm{ab}}$
\end{tabular} & $\begin{array}{c}7.78 \\
\pm 0.16^{\mathrm{bc}}\end{array}$ & $\begin{array}{c}0.60 \\
\pm 0.01^{\mathrm{a}}\end{array}$ & $\begin{array}{c}0.44 \\
\pm 0.01^{\mathrm{abc}}\end{array}$ & $\begin{array}{c}0.33 \\
\pm 0.02^{\mathrm{c}}\end{array}$ & $\begin{array}{c}0.15 \\
\pm 0.01^{\mathrm{ab}}\end{array}$ & $\begin{array}{c}0.02 \\
\pm 0.00^{\mathrm{a}}\end{array}$ \\
\hline 7 & $\begin{array}{c}0.01 \\
\pm 0.00^{\mathrm{a}}\end{array}$ & $\begin{array}{l}11.45 \\
\pm 0.05^{\text {cd }}\end{array}$ & $\begin{array}{c}0.72 \\
\pm 0.00^{\mathrm{d}}\end{array}$ & $\begin{array}{c}0.09 \\
\pm 0.01^{\mathrm{bc}}\end{array}$ & $\begin{array}{c}0.13 \\
\pm 0.00^{\mathrm{b}}\end{array}$ & $\begin{array}{c}3.23 \\
\pm 0.04^{\text {acd }}\end{array}$ & $\begin{array}{l}75.95 \\
\pm 0.20^{\text {de }}\end{array}$ & $\begin{array}{c}6.89 \\
\pm 0.11^{\mathrm{a}}\end{array}$ & $\begin{array}{c}0.61 \\
\pm 0.01^{\mathrm{a}}\end{array}$ & $\begin{array}{c}0.45 \\
\pm 0.00^{\mathrm{abc}}\end{array}$ & $\begin{array}{c}0.30 \\
\pm 0.01^{\mathrm{abc}}\end{array}$ & $\begin{array}{c}0.15 \\
\pm 0.01^{\mathrm{ab}}\end{array}$ & $\begin{array}{c}0.02 \\
\pm 0.01^{\mathrm{a}}\end{array}$ \\
\hline 8 & $\begin{array}{c}0.01 \\
\pm 0.00^{\mathrm{a}}\end{array}$ & $\begin{array}{l}15.02 \\
\pm 0.07^{\mathrm{g}}\end{array}$ & $\begin{array}{c}1.56 \\
\pm 0.01^{\mathrm{e}}\end{array}$ & $\begin{array}{c}0.05 \\
\pm 0.00^{\mathrm{a}}\end{array}$ & $\begin{array}{c}0.08 \\
\pm 0.00^{\mathrm{a}}\end{array}$ & $\begin{array}{c}2.82 \\
\pm 0.02^{\mathrm{ab}}\end{array}$ & $\begin{array}{l}66.52 \\
\pm 0.33^{\text {f }}\end{array}$ & $\begin{array}{l}12.45 \\
\pm 0.20^{\mathrm{e}}\end{array}$ & $\begin{array}{c}0.56 \\
\pm 0.00^{\mathrm{ab}}\end{array}$ & $\begin{array}{c}0.49 \\
\pm 0.04^{\mathrm{c}}\end{array}$ & $\begin{array}{c}0.26 \\
\pm 0.01^{\mathrm{ab}}\end{array}$ & $\begin{array}{c}0.15 \\
\pm 0.01^{\mathrm{ab}}\end{array}$ & $\begin{array}{c}0.03 \\
\pm 0.01^{\mathrm{a}}\end{array}$ \\
\hline 9 & $\begin{array}{c}0.01 \\
\pm 0.00^{\mathrm{a}}\end{array}$ & $\begin{array}{l}11.77 \\
\pm 0.28^{\text {cde }}\end{array}$ & $\begin{array}{c}0.85 \\
\pm 0.03^{\text {abd }}\end{array}$ & $\begin{array}{c}0.11 \\
\pm 0.00^{\mathrm{c}}\end{array}$ & $\begin{array}{c}0.18 \\
\pm 0.00^{\mathrm{d}}\end{array}$ & $\begin{array}{c}3.23 \\
\pm 0.04^{\text {acd }}\end{array}$ & $\begin{array}{l}73.71 \\
\pm 0.66^{\text {ab }}\end{array}$ & $\begin{array}{r}8.53 \\
\pm 0.37^{\mathrm{c}}\end{array}$ & $\begin{array}{c}0.63 \\
\pm 0.01^{\mathrm{a}}\end{array}$ & $\begin{array}{c}0.45 \\
\pm 0.00^{\mathrm{abc}}\end{array}$ & $\begin{array}{c}0.34 \\
\pm 0.02^{\mathrm{c}}\end{array}$ & $\begin{array}{c}0.16 \\
\pm 0.01^{\mathrm{ab}}\end{array}$ & $\begin{array}{c}0.02 \\
\pm 0.00^{\mathrm{a}}\end{array}$ \\
\hline 10 & $\begin{array}{c}0.02 \\
\pm 0.01^{\mathrm{a}}\end{array}$ & $\begin{array}{l}11.94 \\
\pm 0.07^{\text {de }}\end{array}$ & $\begin{array}{c}0.91 \\
\pm 0.03^{\text {adcd }}\end{array}$ & $\begin{array}{c}0.05 \\
\pm 0.00^{\mathrm{a}}\end{array}$ & $\begin{array}{c}0.09 \\
\pm 0.00^{\mathrm{a}}\end{array}$ & $\begin{array}{c}3.75 \\
\pm 0.01^{\mathrm{d}}\end{array}$ & $\begin{array}{l}77.08 \\
\pm 0.09 \mathrm{a}\end{array}$ & $\begin{array}{c}4.73 \\
\pm 0.04^{\mathrm{d}}\end{array}$ & $\begin{array}{c}0.57 \\
\pm 0.01^{\mathrm{ab}}\end{array}$ & $\begin{array}{c}0.42 \\
\pm 0.01^{\mathrm{ab}}\end{array}$ & $\begin{array}{c}0.26 \\
\pm 0.02^{\mathrm{ab}}\end{array}$ & $\begin{array}{c}0.16 \\
\pm 0.02^{\mathrm{ab}}\end{array}$ & $\begin{array}{c}0.02 \\
\pm 0.00^{\mathrm{a}}\end{array}$ \\
\hline 11 & $\begin{array}{c}0.03 \\
\pm 0.01^{\mathrm{a}}\end{array}$ & $\begin{array}{l}11.33 \\
\pm 0.03^{\mathrm{c}}\end{array}$ & $\begin{array}{c}0.81 \\
\pm 0.01^{\text {ad }}\end{array}$ & $\begin{array}{c}0.08 \\
\pm 0.03^{\mathrm{abc}}\end{array}$ & $\begin{array}{c}0.09 \\
\pm 0.00^{\mathrm{a}}\end{array}$ & $\begin{array}{c}2.51 \\
\pm 0.03^{\mathrm{b}}\end{array}$ & \begin{tabular}{|l|}
73.95 \\
$\pm 0.02^{\mathrm{ab}}$
\end{tabular} & $\begin{array}{c}9.78 \\
\pm 0.04^{\text {e }}\end{array}$ & $\begin{array}{c}0.34 \\
\pm 0.21^{\mathrm{b}}\end{array}$ & $\begin{array}{c}0.48 \\
\pm 0.04^{\mathrm{bc}}\end{array}$ & $\begin{array}{c}0.33 \\
\pm 0.02^{\mathrm{c}}\end{array}$ & $\begin{array}{c}0.20 \\
\pm 0.03^{\mathrm{b}}\end{array}$ & $\begin{array}{c}0.03 \\
\pm 0.01^{\mathrm{a}}\end{array}$ \\
\hline $\begin{array}{l}\text { EU Com- } \\
\text { mission } \\
\text { Regulation } \\
\text { No. 1348/ } \\
2013 \\
\text { Rozporzą- } \\
\text { dzenie KE } \\
\text { nr 1348/ } \\
2013\end{array}$ & $\leq 0.03$ & $\begin{array}{l}7.50- \\
20.00\end{array}$ & $\begin{array}{l}0.30- \\
3.50\end{array}$ & $\leq 0.30$ & $\leq 0.30$ & $\begin{array}{l}0.50- \\
5.00\end{array}$ & $\begin{array}{l}55.00- \\
83.00\end{array}$ & $\begin{array}{l}3.50- \\
21.00\end{array}$ & $\leq 1.00$ & $\leq 0.60$ & $\leq 0.40$ & $\begin{array}{l}\leq 0.20 \\
\text { EVOO } \\
\leq 0.3 \\
\text { pomace } \\
\text { wytłoki }\end{array}$ & $\leq 0.20$ \\
\hline
\end{tabular}

Values within columns designated with different letters are statistically significantly different at the level of $\alpha \leq 0.05$. Wartości w kolumnach oznaczone różnymi literami są statystycznie istotnie różne na poziomie $\alpha \leq 0,05$.

\section{Sterols composition}

All the test oils were characterized by a high amount of $\beta$-sitosterol, which ranged from 93.15 to $95.08 \%$ of the sterol fraction and should be not less than $93.0 \%$ (Table 2). Campesterol content should not exceed $4.00 \%$. Only three of olive samples: Nos. 1, 4 and 9 fulfilled this condition. The biggest exceeding of this limit occurred in olive pomace oil. The content of stigmasterol ranged from 0.55 to $1.82 \%$. The Regulation suggests that it should not exceed the content of campesterol. In all the samples $\Delta 7$-stigmasterol was detected and ranged from 0.14 to $0.50 \%$ and did not exceed the limits stated in the Reg- 
Derewiaka, D., Łuczak, A., Reder, M. (2016). Detection of adulteration of extra virgin olive oils available on the Polish market. Nauka Przyr. Technol., 10, 4, \#47. DOI: http://dx.doi.org/10.17306/J.NPT.2016.4.47

Table 2. Sterols composition in selected olive oils

Tabela 2. Skład steroli w wybranych oliwach z oliwek

\begin{tabular}{|c|c|c|c|c|c|c|c|}
\hline $\begin{array}{l}\text { Product } \\
\text { code } \\
\text { Kod } \\
\text { produktu }\end{array}$ & $\begin{array}{c}\text { Cholesterol } \\
(\%)\end{array}$ & $\begin{array}{l}\text { Brassica- } \\
\text { sterol } \\
\text { Brassika- } \\
\text { sterol } \\
(\%)\end{array}$ & $\begin{array}{c}\text { Campesterol } \\
\text { Kampesterol } \\
(\%)\end{array}$ & $\begin{array}{c}\text { Stigmasterol } \\
\text { Stygmasterol } \\
(\%)\end{array}$ & $\begin{array}{c}\beta \text {-Sitosterol } \\
(\%)\end{array}$ & $\begin{array}{c}\Delta 7 \text {-Stigma- } \\
\text { sterol } \\
\Delta 7 \text {-Stygma- } \\
\text { sterol } \\
(\%)\end{array}$ & $\begin{array}{c}\text { Total sterols } \\
\text { Sterole } \\
\text { ogółem } \\
(\mathrm{mg} / \mathrm{kg})\end{array}$ \\
\hline 1 & $\begin{array}{c}0.36 \\
\pm 0.12^{\mathrm{abc}}\end{array}$ & $\begin{array}{r}0.09 \\
\pm 0.03^{\mathrm{a}}\end{array}$ & $\begin{array}{c}3.71 \\
\pm 0.31^{\mathrm{a}}\end{array}$ & $\begin{array}{c}0.55 \\
\pm 0.02^{\mathrm{a}}\end{array}$ & $\begin{array}{l}95.02 \\
\pm 0.63^{\mathrm{a}}\end{array}$ & $\begin{array}{c}0.27 \\
\pm 0.00^{\mathrm{ab}}\end{array}$ & $\begin{array}{l}1058 \\
\pm 55^{\mathrm{ab}}\end{array}$ \\
\hline 2 & $\begin{array}{r}0.46 \\
\pm 0.14^{\mathrm{a}}\end{array}$ & $\begin{aligned} & 0.16 \\
\pm & 0.08^{\mathrm{a}}\end{aligned}$ & $\begin{aligned} & 4.16 \\
\pm & 0.04^{\mathrm{a}}\end{aligned}$ & $\begin{array}{c}1.03 \\
\pm 0.18^{\text {abc }}\end{array}$ & $\begin{array}{l}94.02 \\
\pm 0.14^{\mathrm{ab}}\end{array}$ & $\begin{array}{c}0.14 \\
\pm 0.10^{\mathrm{a}}\end{array}$ & $\begin{array}{l}1170 \\
\pm 42^{\mathrm{b}}\end{array}$ \\
\hline 3 & $\begin{array}{c}0.38 \\
\pm 0.12^{\mathrm{ac}}\end{array}$ & $\begin{array}{c}0.08 \\
\pm 0.04^{\mathrm{a}}\end{array}$ & $\begin{aligned} & 4.11 \\
\pm & 0.04^{\mathrm{a}}\end{aligned}$ & $\begin{array}{c}0.80 \\
\pm 0.08^{\mathrm{ab}}\end{array}$ & $\begin{array}{l}94.14 \\
\pm 0.18^{\mathrm{ab}}\end{array}$ & $\begin{array}{c}0.48 \\
\pm 0.10^{\mathrm{ab}}\end{array}$ & $\begin{array}{l}1031 \\
\quad \pm 28^{\mathrm{ab}}\end{array}$ \\
\hline 4 & $\begin{array}{c}0.34 \\
\pm 0.03^{\mathrm{abc}}\end{array}$ & $\begin{array}{c}0.08 \\
\pm 0.07^{\mathrm{a}}\end{array}$ & $\begin{aligned} & 3.87 \\
\pm & 0.17^{\mathrm{a}}\end{aligned}$ & $\begin{array}{c}1.10 \\
\pm 0.23^{\mathrm{ab}}\end{array}$ & $\begin{array}{l}94.11 \\
\pm 0.41^{\mathrm{ab}}\end{array}$ & $\begin{array}{c}0.50 \\
\pm 0.05^{\mathrm{b}}\end{array}$ & $\begin{array}{r}1066 \\
\quad \pm 8^{\mathrm{ab}}\end{array}$ \\
\hline 5 & $\begin{array}{c}0.36 \\
\pm 0.12^{\text {abc }}\end{array}$ & $\begin{array}{r}0.09 \\
\pm 0.03^{\mathrm{a}}\end{array}$ & 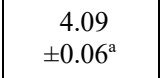 & $\begin{aligned} & 0.95 \\
\pm & 0.17^{\text {abc }}\end{aligned}$ & $\begin{array}{l}94.26 \\
\pm 0.64^{\mathrm{bc}}\end{array}$ & $\begin{array}{c}0.45 \\
\pm 0.20^{\mathrm{ab}}\end{array}$ & $\begin{array}{l}1034 \\
\quad \pm 27^{\mathrm{abc}}\end{array}$ \\
\hline 6 & $\begin{aligned} & 0.31 \\
\pm & 0.09^{\mathrm{abc}}\end{aligned}$ & $\begin{array}{c}0.10 \\
\pm 0.02^{\mathrm{a}}\end{array}$ & $\begin{array}{r}4.29 \\
\pm 0.19^{\mathrm{a}}\end{array}$ & $\begin{aligned} & 1.55 \\
\pm & 0.13^{\mathrm{ab}}\end{aligned}$ & $\begin{array}{l}94.15 \\
\pm 0.52^{\mathrm{ab}}\end{array}$ & $\begin{array}{c}0.27 \\
\pm 0.01^{\mathrm{ab}}\end{array}$ & $\begin{array}{l}1011 \\
\quad \pm 10^{\mathrm{ac}}\end{array}$ \\
\hline 7 & $\begin{array}{c}0.20 \\
\pm 0.01^{\mathrm{bc}}\end{array}$ & $\begin{array}{c}0.10 \\
\pm 0.06^{\mathrm{a}}\end{array}$ & $\begin{aligned} & 4.25 \\
\pm & 0.16^{\mathrm{a}}\end{aligned}$ & $\begin{array}{c}0.98 \\
\pm 0.04^{\text {abc }}\end{array}$ & $\begin{array}{l}94.16 \\
\pm 0.23^{\mathrm{ab}}\end{array}$ & $\begin{array}{c}0.30 \\
\pm 0.08^{\mathrm{ab}}\end{array}$ & $\begin{array}{l}1093 \\
\pm 49^{\mathrm{ab}}\end{array}$ \\
\hline 8 & $\begin{array}{c}0.22 \\
\pm 0.02^{\text {abc }}\end{array}$ & $\begin{array}{c}0.07 \\
\pm 0.01^{\mathrm{a}}\end{array}$ & 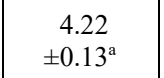 & $\begin{array}{c}0.90 \\
\pm 0.02^{\text {abc }}\end{array}$ & $\begin{array}{l}94.07 \\
\pm 0.00^{\mathrm{abc}}\end{array}$ & $\begin{array}{c}0.48 \\
\pm 0.12^{\mathrm{ab}}\end{array}$ & $\begin{array}{l}1303 \\
\pm 50^{\mathrm{d}}\end{array}$ \\
\hline 9 & $\begin{array}{c}0.07 \\
\pm 0.02^{\mathrm{b}}\end{array}$ & $\begin{array}{c}0.10 \\
\pm 0.01^{\mathrm{a}}\end{array}$ & $\begin{array}{r}3.64 \\
\pm 0.17^{\mathrm{a}}\end{array}$ & $\begin{array}{c}0.87 \\
\pm 0.14^{\mathrm{ab}}\end{array}$ & $\begin{array}{l}95.08 \\
\pm 0.07^{\mathrm{a}}\end{array}$ & $\begin{array}{c}0.24 \\
\pm 0.06^{\mathrm{ab}}\end{array}$ & $\begin{array}{l}1091 \\
\quad \pm 52^{\text {abd }}\end{array}$ \\
\hline 10 & $\begin{array}{c}0.18 \\
\pm 0.01^{\mathrm{ac}}\end{array}$ & $\begin{array}{c}0.09 \\
\pm 0.02^{\mathrm{a}}\end{array}$ & $\begin{array}{c}4.06 \\
\pm 0.00^{\mathrm{a}}\end{array}$ & $\begin{array}{c}1.17 \\
\pm 0.06^{\mathrm{bc}}\end{array}$ & $\begin{array}{l}94.04 \\
\pm 0.11^{\mathrm{abc}}\end{array}$ & $\begin{array}{c}0.48 \\
\pm 0.05^{\mathrm{ab}}\end{array}$ & $\begin{array}{l}1032 \\
\quad \pm 32^{\mathrm{ab}}\end{array}$ \\
\hline 11 & $\begin{array}{c}0.44 \\
\pm 0.05^{\mathrm{a}}\end{array}$ & $\begin{array}{c}0.18 \\
\pm 0.06^{\mathrm{a}}\end{array}$ & $\begin{array}{c}5.02 \\
\pm 0.37^{\mathrm{a}}\end{array}$ & $\begin{array}{c}1.82 \\
\pm 0.09^{\mathrm{c}}\end{array}$ & $\begin{array}{l}93.15 \\
\pm 0.60^{\mathrm{bc}}\end{array}$ & $\begin{array}{c}0.36 \\
\pm 0.11^{\mathrm{ab}}\end{array}$ & $\begin{array}{r}1292 \\
\pm 18^{\mathrm{d}}\end{array}$ \\
\hline $\begin{array}{l}\text { EU Com- } \\
\text { mission } \\
\text { Regulation } \\
\text { No. 1348/ } \\
2013 \\
\text { Rozporzą- } \\
\text { dzenie KE } \\
\text { nr 1348/ } \\
2013\end{array}$ & $\leq 0.5$ & $\begin{array}{l}\leq 0.1 \text { for } / \mathrm{dla} \\
\text { EVOO } \\
\leq 0.2 \text { for } / \mathrm{dla} \\
\text { VLOO }\end{array}$ & $\leq 4.0$ & $\begin{array}{l}\leq \text { camp } \\
\leq \text { kamp }\end{array}$ & $\geq 93.0$ & $\leq 0.5$ & $\begin{array}{l}\geq 1000 \\
\text { for/dla } \\
\text { EVOO } \\
\geq 1600 \\
\text { for/dla } \\
\text { VLOO }\end{array}$ \\
\hline
\end{tabular}

$* \Delta$-5,23-Stigmastadienol+clerosterol $+\beta$-sitosterol + sitostanol $+\Delta$-5-avenasterol $+\Delta-5,24$-stigmastadienol.

Values within columns designated with different letters are statistically significantly different at the level of $\alpha \leq 0.05$.

$* \Delta-5,23$-Stygmastadienol+clerosterol $+\beta$-sitosterol + sitostanol $+\Delta$-5-avenasterol $+\Delta-5,24$-stygmastadienol.

Wartości w kolumnach oznaczone różnymi literami są statystycznie istotnie różne na poziomie $\alpha \leq 0,05$.

ulation $-0.50 \%$. Cholesterol and brassicasterol have the smallest share in the composition of the sterol fraction, which was $0.07-0.46 \%$ and $0.07-0.18 \%$, respectively. The 
Derewiaka, D., Łuczak, A., Reder, M. (2016). Detection of adulteration of extra virgin olive oils available on the Polish market. Nauka Przyr. Technol., 10, 4, \#47. DOI: http://dx.doi.org/10.17306/J.NPT.2016.4.47

Regulation No 1348/2013 (Commission..., 2013b) establishes that cholesterol content in EVOO should not exceed $0.50 \%$ and brassicasterol $-0.20 \%$. Moreover, the total content of sterols in EVOO should be higher than $1000 \mathrm{mg}$ per $1 \mathrm{~kg}$ of oil and $1600 \mathrm{mg}$ per $1 \mathrm{~kg}$ in olive pomace oil. In samples Nos. 1, 3, 5, 6, 10 the total content of sterols was near $1000 \mathrm{mg} / \mathrm{kg}$. In other EVOO's it was higher than $1000 \mathrm{mg} / \mathrm{kg}$, but the product met the conditions of the Regulation, because the total content of sterols in olive pomace oils was lower than $1600 \mathrm{mg} / \mathrm{kg}$. In Table 3 the percentage of sum of erythrodiol and uvaol in overall sterols fraction was presented. The level of the compounds can indicate adulteration of high quality olive oils addition of oils of the following types: olive pomace oils, crude and refined olive pomace oil. According to the Regulation No 1348/2013 (Commission..., 2013b) the level of erythrodiol and uvaol in the sterols fraction of EVOO, VOO, lampante, refined olive oil and olive oil composed of refined and virgin olive oils should be $\leq 4.5 \%$. In samples of EVOO Nos. 2-10 the total erythrodiol and uvaol content ranged from $1.70 \%$ to $3.73 \%$, and did not exceed the limit established by the Regulation. The obtained results of the determination of erythrodiol and uvaol in EVOO No 1 and olive pomace oil No $11(4.70 \%)$ differ statistically significantly from the remaining olive oil results and can indicate that EVOO No 1 might have been adulterated by an addition of olive pomace oils, crude, refined olive pomace oil or other oils (Fig. 1). Cunha et al. (2006) determined $\beta$-sitosterol in Portuguese olive oils and its content was between $49.00-187.00 \mathrm{mg}$ in $100 \mathrm{~g}$. Another abundant phytosterol in sterols

Table 3. Erythrodiol and uvaol content in selected olive oils (\%)

Tabela 3. Zawartość erytrodiolu i uwaolu w wybranych oliwach z oliwek (\%)

\begin{tabular}{|c|c|}
\hline $\begin{array}{c}\text { Product code } \\
\text { Kod produktu }\end{array}$ & $\begin{array}{c}\text { Content } \\
\text { Zawartość }\end{array}$ \\
\hline 1 & $5.32 \pm 0.00^{\mathrm{f}}$ \\
2 & $2.08 \pm 0.15^{\mathrm{ac}}$ \\
4 & $2.51 \pm 0.19^{\mathrm{ab}}$ \\
5 & $3.39 \pm 0.17^{\mathrm{de}}$ \\
6 & $2.29 \pm 0.33^{\mathrm{abc}}$ \\
7 & $3.73 \pm 0.14^{\mathrm{e}}$ \\
8 & $2.96 \pm 0.22^{\mathrm{bd}}$ \\
9 & $2.34 \pm 0.11^{\mathrm{abc}}$ \\
10 & $2.55 \pm 0.09^{\mathrm{ab}}$ \\
11 & $1.70 \pm 0.27^{\mathrm{c}}$ \\
\hline
\end{tabular}

Values within columns designated with different letters are statistically significantly different at the level of $\alpha \leq 0.05$.

Wartości w kolumnach oznaczone różnymi literami są statystycznie istotnie różne na poziomie $\alpha \leq 0,05$. 
Derewiaka, D., Łuczak, A., Reder, M. (2016). Detection of adulteration of extra virgin olive oils available on the Polish market. Nauka Przyr. Technol., 10, 4, \#47. DOI: http://dx.doi.org/10.17306/J.NPT.2016.4.47

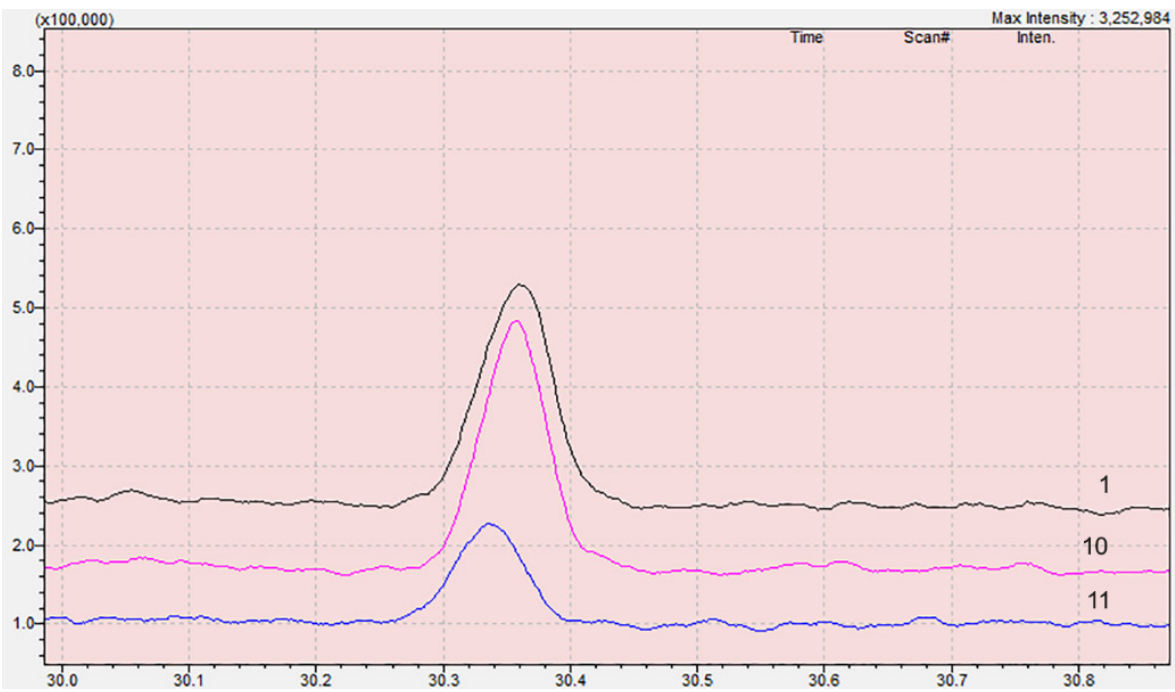

Fig. 1. Chromatogram of erythrodiol and uvaol in extra virgin olive oils Nos. 1, 10 and olive pomace oil No 11

Rys. 1. Chromatogram erytrodiolu i uwaolu w oliwach extra virgin $\mathrm{nr} 1,10$ oraz w oliwie z wytłoków nr 11

fraction of the investigated olive oils was 24-methylene cycloartenol and its content ranged between 8.55 (sample No 1) and 28.13 (sample No 8) $\mathrm{mg}$ in 100 g. Sakouhi et al. (2010) investigated that content of 24-methylene cycloartenol in Tunisian olive oils was higher reaching $14.60-17.30 \mathrm{mg}$ in $100 \mathrm{~g}$. The content of $\Delta 5$-avenasterol in the analysed olives was between 5.94 (sample No 9) and 13.17 (sample No 1) mg in $100 \mathrm{~g}$ and was not so varied as it was described by Aparicio and Aparicio-Ruiz (2000) - 3.00 $26.50 \mathrm{mg}$ in $100 \mathrm{~g}$. Sakouhi et al. (2010) found that the level of cycloartenol in olive oils can reach $75 \mathrm{mg}$ in $100 \mathrm{~g}$. Lukic et al. (2013) determined almost the same range of campesterol content (2.80-3.36 mg in $100 \mathrm{~g})$ in Croatian olives.

\section{Determination of extinction coefficient $K_{232}, K_{270}$ and $\Delta K$}

According to the Regulation No 1348/2013 (Commission..., 2013b) extinction coefficients $\mathrm{K}_{232}$ and $\mathrm{K}_{270}$ for EVOO should be $\leq 2.5$ and $\leq 0.22$, respectively and $\Delta \mathrm{K}$ should be $\leq 0.01$. In the olive pomace oil sample the investigated coefficients of $\mathrm{K}_{232}$ and $\mathrm{K}_{270}$ were 2.96 and 1.05 , respectively, and $\Delta \mathrm{K}$ was 0.077 . These results were higher than the results presented for EVOO (Table 4). Among all the investigated EVOO samples only two (No 1 and No 4) had higher values of $\mathrm{K}_{232}$ and $\mathrm{K}_{270}$ than the limits given in the Regulation No 1348/2013 (Commission..., 2013b), which may indicate adulteration of these samples. The value of coefficient $K_{232}$ for sample No 1 was 2.54. The value of coefficient $K_{270}$ was 0.25 for samples No 1 and No 4. Other EVOO sample coefficient values of $\mathrm{K}_{232}$ and $\mathrm{K}_{270}$ complied with the requirements of the Regulation No 1348/2013 (Commission..., 2013b) (Table 4). Similar results of extinction coefficient obtained Rigane et al. (2013) and described that the extinction coefficients for Tunisian EVOO ranged from 1.78 to 2.35 for 
Derewiaka, D., Łuczak, A., Reder, M. (2016). Detection of adulteration of extra virgin olive oils available on the Polish market. Nauka Przyr. Technol., 10, 4, \#47. DOI: http://dx.doi.org/10.17306/J.NPT.2016.4.47

Table 4. Values of extinction coefficients $\left(\mathrm{K}_{232}\right.$ and $\left.\mathrm{K}_{270}\right)$ and the volatility of specific optical density $(\Delta \mathrm{K})$

Tabela 4. Wartości współczynników ekstynkcji $\left(\mathrm{K}_{232}\right.$ i $\left.\mathrm{K}_{270}\right)$ oraz zmienności właściwej gęstości optycznej $(\Delta \mathrm{K})$

\begin{tabular}{|c|c|c|c|}
\hline $\begin{array}{c}\text { Product code } \\
\text { Kod produktu }\end{array}$ & $\mathrm{K}_{232}$ & $\mathrm{~K}_{270}$ & $\Delta \mathrm{K}$ \\
\hline 1 & $2.54 \pm 0.02^{\mathrm{a}}$ & $0.25 \pm 0.01^{\mathrm{b}}$ & 0.007 \\
2 & $2.34 \pm 0.11^{\mathrm{bc}}$ & $0.21 \pm 0.01^{\mathrm{a}}$ & 0.003 \\
3 & $2.20 \pm 0.03^{\mathrm{cd}}$ & $0.20 \pm 0.01^{\mathrm{a}}$ & 0.007 \\
4 & $2.52 \pm 0.02^{\mathrm{a}}$ & $0.25 \pm 0.01^{\mathrm{b}}$ & 0.004 \\
5 & $2.49 \pm 0.01^{\mathrm{ab}}$ & $0.21 \pm 0.01^{\mathrm{a}}$ & 0.003 \\
6 & $2.28 \pm 0.01^{\mathrm{abc}}$ & $0.20 \pm 0.02^{\mathrm{a}}$ & 0.006 \\
7 & $2.48 \pm 0.03^{\mathrm{ab}}$ & $0.21 \pm 0.01^{\mathrm{a}}$ & 0.001 \\
8 & $2.46 \pm 0.02^{\mathrm{ab}}$ & $0.22 \pm 0.01^{\mathrm{ab}}$ & 0.002 \\
9 & $2.48 \pm 0.04^{\mathrm{ab}}$ & $0.20 \pm 0.02^{\mathrm{a}}$ & 0.005 \\
10 & $2.07 \pm 0.13^{\mathrm{d}}$ & $0.19 \pm 0.01^{\mathrm{a}}$ & 0.009 \\
11 & $2.96 \pm 0.05^{\mathrm{e}}$ & $1.05 \pm 0.02^{\mathrm{c}}$ & 0.077 \\
\hline
\end{tabular}

Values within columns designated with different letters are statistically significantly different at the level of $\alpha \leq 0.05$.

Wartości w kolumnach oznaczone różnymi literami są statystycznie istotnie różne na poziomie $\alpha \leq 0,05$.

$\mathrm{K}_{232}$, and from 0.18 to 0.19 for $\mathrm{K}_{270}$. Krichène et al. (2010) obtained significantly lower values of those coefficients in Italian EVOO: 1.54-2.01 for $\mathrm{K}_{232}$ and $0.09-0.13$ for $\mathrm{K}_{270}$ what might have been due to the high quality of the tested olives.

\section{Conclusions}

Our study on the authentication of extra virgin olive oils available on the Polish market was based on analyses of some parameters (fatty acids profile, sterols content and extinction coefficient $\mathrm{K}_{232}, \mathrm{~K}_{270}$ and $\Delta \mathrm{K}$ ), which values are described in the Commission Implementing Regulation (EU) No 1348/2013 of 16 December 2013 amending Regulation (EEC) No 2568/91 on the characteristics of olive oil and olive-residue oil and on the relevant methods of analysis (2013b). The Regulation provides the values of fatty acids profiles, sterols content and extinction coefficient $\mathrm{K}_{232}, \mathrm{~K}_{270}$ and $\Delta \mathrm{K}$, which are adequate for extra virgin olive oils and other categories of oil, e.g. olive pomace oils. The results of fatty acids composition of analysed extra virgin olive oil did not show any abnormalities of standards values specified by the Regulation. On the basis of the sterols content determination it was proven that extra virgin olive oil No 1 was falsified, because the erythrodiol and uvaol content should be $\leq 4.5 \%$ and the result was 
Derewiaka, D., Łuczak, A., Reder, M. (2016). Detection of adulteration of extra virgin olive oils available on the Polish market. Nauka Przyr. Technol., 10, 4, \#47. DOI: http://dx.doi.org/10.17306/J.NPT.2016.4.47

$5.32 \%$. Two extra virgin olive oil samples included in the current analysis showed levels of $\mathrm{K}_{232}$ and/or $\mathrm{K}_{270}$ values higher than expected for this category of olive oil or other oils presented in Regulation No 1348/2013 (Commission..., 2013b). The aim of the study was reached, because our research proves that sometimes consumers are misinformed and some of food manufacturers want to sell lower quality products as high-quality products, like extra virgin olive oil No 1 available on the Polish market. There is a need to constantly monitor the quality of foodstuffs and provide consumers with access to reliable information on food products.

\section{References}

Aparicio, R., Aparicio-Ruiz, R. (2000). Authentication of vegetable oils by chromatographic techniques. J. Chromatogr., 881, 93-104. DOI: 10.1016/S0021-9673(00)00355-1

Aparicio, R., Morales, M. T., Aparicio-Ruiz, R., Tena, N., García-González, D. L. (2013). Authenticity of olive oil: mapping and comparing official methods and promising alternatives. Food Res. Int., 54, 2, 2025-2038. DOI: 10.1016/j.foodres.2013.07.039

Christopoulou, E., Lazaraki, M., Komaitis, M., Kaselimis, K. (2004). Effectiveness of determinations of fatty acids and triglycerides for the detection of adulteration of olive oils with vegetable oils. Food Chem., 84, 463-474. DOI: 10.1016/S0308-8146(03)00273-5

Commission Implementing Regulation (EU) No 299/2013 of 26 March 2013 amending Regulation (EEC) No 2568/91 on the characteristics of olive oil and olive-residue oil and on the relevant methods of analysis. (2013a). Off. J. EU, L, 90, 52-70.

Commission Implementing Regulation (EU) No 1348/2013 of 16 December 2013 amending Regulation (EEC) No 2568/91 on the characteristics of olive oil and olive-residue oil and on the relevant methods of analysis. (2013b). Off. J. EU, L, 338, 31-67.

Commission Regulation (EEC) No 2568/91 of 11 July 1991 on the characteristics of olive oil and olive-residue oil and on the relevant methods of analysis. (1991). Off. J. Eur. Commun., L, $248,34,1-83$.

Cunha, S. S., Fernandes, O. J., Oliveira, M. (2006). Quantification of free and esterified sterols in Portuguese olive oils by solid-phase extraction and gas chromatography-mass spectrometry. J. Chromatogr., 1128, 220-227. DOI: 10.1016/j.chroma.2006.06.039

Derewiaka, D., Waligóra-Okraska, P., Maszewska, M., Zaręba, D. (2014). Charakterystyka składu wybranych tłuszczów do smarowania pieczywa występujących na rynku warszawskim. Towarozn. Probl. Jakości, 39, 2, 56-67.

Garcia, R., Martins, N., Cabrita, M. J. (2013). Putative markers of adulteration of extra virgin olive oil with refined olive oil: prospects and limitations. Food Res. Int., 54, 2, 2039-2044. DOI: $10.1016 /$ j.foodres.2013.05.008

Krichène, D., Allalout, A., Salvador, M. D., Fregapane, G., Zarrouk, M. (2010). Fatty acids, volatiles, sterols and triterpenic alcohols of six monovarietal Tunisian virgin olive oils. Eur. J. Lipid Sci. Technol., 112, 3, 400-409. DOI: 10.1002/ejlt.200900095

Lukic, M., Lukic, I., Krapac, M., Sladonja, B., Pilizota, V. (2013). Sterols and triterpene diols in olive oil as indicators of variety and degree of ripening. Food Chem., 136, 251-258. DOI: 10.1016/j.foodchem.2012.08.005

Rigane, G., Ayadi, M., Boukhris, M., Sayadi, S., Bouaziz, M. (2013). Characterisation and phenolic profiles of two rare olive oils from southern Tunisia: Dhokar and Gemri-Dhokar cultivars. J. Sci. Food Agric., 93, 3, 527-534. DOI: 10.1002/jsfa.5815

Sakouhi, F., Absalon, C., Flamini, G., Cioni, P. L., Kallel, H., Boukhchina, S. (2010). Lipid components of olive oil from Tunisian Cv. Sayali: characterization and authenticity. C. R. Biol., 333, 9, 642-648. DOI: 10.1016/j.crvi.2010.05.001 
Derewiaka, D., Łuczak, A., Reder, M. (2016). Detection of adulteration of extra virgin olive oils available on the Polish market. Nauka Przyr. Technol., 10, 4, \#47. DOI: http://dx.doi.org/10.17306/J.NPT.2016.4.47

van Wetten, I. A., van Herwaarden, A. W., Splinter, R., Boerrigter-Eenling, R., van Ruth, S. M. (2015). Detection of sunflower oil in extra virgin olive oil by fast differential scanning calorimetry. Thermochim. Acta, 603, 237-243. DOI: 10.1016/j.tca.2014.11.030

\section{WYKRYWANIE ZAFAŁSZOWAŃ OLIWY Z OLIWEK EXTRA VIRGIN DOSTĘPNEJ NA RYNKU POLSKIM}

\section{Streszczenie}

Wstęp. Oliwy extra virgin są cennym i zdrowym, cenionym przez konsumentów produktem. Celem niniejszej pracy była próba wykrycia zafałszowań oliwy z oliwek dostępnej na rynku polskim (pochodzącej z Grecji, Włoch i Hiszpanii) zgodnie z Rozporządzeniem Komisji Europejskiej nr 1348/2013.

Material i metody. W 10 wybranych oliwach extra virgin dostępnych na rynku polskim zbadano skład kwasów tłuszczowych, skład i zawartość steroli (w tym erytrodiolu i uwaolu) z użyciem chromatografii gazowej sprzężonej ze spektrometrem mas oraz wartości współczynników ekstynkcji $\mathrm{K}_{232}$ i K 270 . Otrzymane wyniki porównano z wymaganiami stawianymi w Rozporządzeniu Komisji Europejskiej nr 1348/2013.

Wyniki. Jedną z najbardziej efektywnych metod wykrywania zafałszowań oliwy z oliwek jest analiza ilościowa i jakościowa steroli. W niektórych spośród badanych oliw extra virgin wykryto nieprawidłowości dotyczące składu, które mogą wskazywać na próbę zafałszowania produktów. Jedna $\mathrm{z}$ opisanych metod może potwierdzić, że dwie próbki oliwy z oliwek extra virgin zostały zafałszowane, oraz można za jej pomocą stwierdzić, czy zostały one zafałszowane poprzez dodanie oliwy z oliwek z wytłoków.

Wnioski. Nasze badania dowodzą, że czasem konsumenci są wprowadzani w błąd, a niektórzy producenci żywności chcą sprzedać produkty o gorszej jakości jako produkty o jakości bardzo dobrej. Istnieje potrzeba ciągłego monitorowania jakości żywności oraz umożliwienia konsumentom dostępu do rzetelnej informacji na temat produktów żywnościowych.

Słowa kluczowe: oliwa z oliwek, kwasy tłuszczowe, sterole, współczynnik ekstynkcji, zafałszowanie

Corresponding address - Adres do korespondencji:

Dorota Derewiaka, Katedra Biotechnologii, Mikrobiologii i Oceny Żywności, Szkoła Główna Gospodarstwa Wiejskiego w Warszawie, ul. Nowoursynowska 159, 02-776 Warszawa, Poland, e-mail:dorota_derewiaka@sggw.pl

Accepted for publication - Zaakceptowano do opublikowania:

22.11.2016

For citation - Do cytowania:

Derewiaka, D., Łuczak, A., Reder, M. (2016). Detection of adulteration of extra virgin olive oils available on the Polish market. Nauka Przyr. Technol., 10, 4, \#47. DOI: http://dx.doi.org/10. 17306/J.NPT.2016.4.47 